\title{
$+-$ \\ INITIAL-BOUNDARY VALUE PROBLEM FOR A TIME-FRACTIONAL SUBDIFFUSION EQUATION ON THE TORUS
}

\author{
RAVSHAN ASHUROV AND OQILA MUHIDDINOVA
}

\begin{abstract}
An initial-boundary value problem for a time-fractional subdiffusion equation with the Riemann-Liouville derivatives on $N$-dimensional torus is considered. Uniqueness and existence of the classical solution of the posed problem are proved by the classical Fourier method. Sufficient conditions for the initial function and for the right-hand side of the equation are indicated, under which the corresponding Fourier series converge absolutely and uniformly. It should be noted, that the condition on the initial function found in this paper is less restrictive than the analogous condition in the case of an equation with derivatives in the sense of Caputo.
\end{abstract}

AMS 2000 Mathematics Subject Classifications : Primary 35R11; Secondary 74S25. Key words: Time-fractional subdiffusion equation, the Riemann-Liouville derivatives, initial-boundary value problem, Fourier method, Liouville spaces.

\section{MAin Result}

The fractional integration of order $\sigma<0$ of the function $h(t)$ defined on $[0, \infty)$ has the form

$$
\partial_{t}^{\sigma} h(t)=\frac{1}{\Gamma(-\rho)} \int_{0}^{t} \frac{h(\xi)}{(t-\xi)^{\sigma+1}} d \xi, \quad t>0,
$$

provided the right-hand side exists. Here $\Gamma(\sigma)$ is Euler's gamma function. Using this definition one can define the Riemann - Liouville fractional derivative of order $\rho, 0<\rho<1$, as (see, for example, 1, p. 14)

$$
\partial_{t}^{\rho} h(t)=\frac{d}{d t} \partial_{t}^{\rho-1} h(t)
$$

If in this definition we interchange differentiation and fractional integration, then we get the definition of the regularized derivative, that is, the definition of the fractional derivative in the sense of Caputo:

$$
D_{t}^{\rho} h(t)=\partial_{t}^{\rho-1} \frac{d}{d t} h(t)
$$

Note that if $\rho=1$, then fractional derivatives coincides with the ordinary classical derivative of the first order: $\partial_{t} h(t)=D_{t} h(t)=\frac{d}{d t} h(t)$.

Let $\mathbb{T}^{N}$ be $N$-dimensional torus: $\mathbb{T}^{N}=(-\pi, \pi]^{N}, N \geq 1$. We define by $C\left(\mathbb{T}^{N}\right)$ and $C^{2}\left(\mathbb{T}^{N}\right)$ a class of $2 \pi$-periodic on each variable $x_{j}$ functions $v(x)$ from $C\left(\mathbb{T}^{N}\right)$ and $C^{2}\left(\mathbb{T}^{N}\right)$ correspondingly. Let $A$ stand for a positive operator, defined on $C^{2}\left(\mathbb{T}^{N}\right)$ and acting as $A v(x)=-\Delta v(x)$, where $\Delta$ is the Laplace operator.

Let $\rho \in(0,1)$ be a constant number. Consider the initial-boundary value problem

$$
\begin{gathered}
\partial_{t}^{\rho} u(x, t)+A u(x, t)=f(x, t), \quad x \in \mathbb{T}^{N}, \quad 0<t \leq T, \\
\lim _{t \rightarrow 0} \partial_{t}^{\rho-1} u(x, t)=\varphi(x), \quad x \in \mathbb{T}^{N},
\end{gathered}
$$

where $f$ and $\varphi$ are given continuous functions.

Definition 1.1. A function $u(x, t)$ with the properties $\partial_{t}^{\rho} u(x, t), A(x, D) u(x, t) \in C\left(\mathbb{T}^{N} \times(0, T]\right)$, $\partial_{t}^{\rho-1} u(x, t) \in C\left(\mathbb{T}^{N} \times[0, T]\right)$ and satisfying the conditions of problem (1.1) - (1.2) is called the solution of the initial-boundary value problem. 
Before formulating the main result, let us introduce some concepts.

It is not hard to see that the closure $\hat{A}$ of operator $A$ in $L_{2}\left(\mathbb{T}^{N}\right)$ is selfadjoint and it has a complete (in $L_{2}\left(\mathbb{T}^{N}\right)$ ) set of eigenfunctions $\left\{\gamma e^{i n x}\right\}, \gamma=\gamma(N)=(2 \pi)^{-N / 2}, n \in \mathbb{Z}^{N}$ and corresponding eigenvalues $|n|^{2}=n_{1}^{2}+n_{2}^{2}+\ldots+n_{N}^{2}$. Therefore, by virtue of J. von Niemann theorem, for any $\tau>0$ one can introduce the power of operator $\hat{A}$ as $\hat{A}^{\tau} g(x)=\sum_{n \in \mathbb{Z}^{N}}|n|^{\tau} g_{n} e^{i n x}$, where $g_{n}$ are Fourier coefficients:

$$
g_{n}=(2 \pi)^{-N} \int_{\mathbb{T}^{N}} g(x) e^{-i n x} d x .
$$

The domain of definition of this operator is defined from the condition $\hat{A}^{\tau} g(x) \in L_{2}\left(\mathbb{T}^{N}\right)$ and has the form

$$
D\left(\hat{A}^{\tau}\right)=\left\{g \in L_{2}\left(\mathbb{T}^{N}\right): \sum_{n \in \mathbb{Z}^{N}}|n|^{2 \tau}\left|g_{n}\right|^{2}<\infty\right\} .
$$

On the other hand, the class of functions $L_{2}\left(\mathbb{T}^{N}\right)$ which for a given fixed number $a>0$ make the norm

$$
\|g\|_{L_{2}^{a}\left(\mathbb{T}^{N}\right)}^{2}=\left\|\sum_{n \in \mathbb{Z}^{N}}\left(1+|n|^{2}\right)^{\frac{a}{2}} g_{n} e^{i n x}\right\|_{L_{2}\left(\mathbb{T}^{N}\right)}^{2}=\sum_{n \in \mathbb{Z}^{N}}\left(1+|n|^{2}\right)^{a}\left|g_{n}\right|^{2}
$$

finite is termed the Liouville class $L_{2}^{a}\left(\mathbb{T}^{N}\right)$. Therefore one has $D\left(\hat{A}^{\tau}\right)=L_{2}^{\tau m}\left(\mathbb{T}^{N}\right)$.

Let $E_{\rho, \mu}$ be the two-parametric Mittag-Leffler function:

$$
E_{\rho, \mu}(t)=\sum_{k=0}^{\infty} \frac{t^{k}}{\Gamma(\rho k+\mu)}
$$

Here is the main result.

Theorem 1.2. Let $a>\frac{N}{2}$ and $\varphi \in C\left(\mathbb{T}^{N}\right) \cap L_{2}^{a-2}\left(\mathbb{T}^{N}\right)$. Moreover, let $f(x, t) \in L_{2}^{a}\left(\mathbb{T}^{N}\right)$ for $0<t \leq T$ and $\left\|t^{1-\rho} f(\cdot, t)\right\|_{L_{2}^{a}\left(\mathbb{T}^{N}\right)}^{2} \in C[0, T]$. Then there exists a solution of initial-boundary value problem (1.1) - (1.2) and it has the form

$$
u(x, t)=\sum_{n \in \mathbb{Z}^{N}}\left[\varphi_{n} t^{\rho-1} E_{\rho, \rho}\left(-|n|^{2} t^{\rho}\right)+\int_{0}^{t} f_{n}(t-\xi) \xi^{\rho-1} E_{\rho, \rho}\left(-|n|^{2} \xi^{\rho}\right) d \xi\right] e^{i n x},
$$

which absolutely and uniformly converges on $x \in \mathbb{T}^{N}$ and for each $t \in(0, T]$, where $\varphi_{n}$ and $f_{n}(t)$ are corresponding Fourier coefficients. Moreover, the series obtained after applying term-wise the operators $\partial_{t}^{\rho}$ and $A$ also converge absolutely and uniformly on $x \in \mathbb{T}^{N}$ and for each $t \in(0, T]$.

Remark 1.3. If $N \leq 3$, then under the conditions of the theorem it suffices to require $\varphi \in$ $C\left(\mathbb{T}^{N}\right)$. Note, when $a>\frac{N}{2}$, according to the Sobolev embedding theorem, all functions in $L_{2}^{a}\left(\mathbb{T}^{N}\right)$ are $2 \pi$-periodic continuous functions. The fulfillment of the inverse inequality $a \leq \frac{N}{2}$, admits the existence of unbounded functions in $L_{2}^{a}\left(\mathbb{T}^{N}\right)$ (see, for example, 17]). Therefore, condition $a>\frac{N}{2}$ for function $f$ of this theorem is not only sufficient for the statement to be hold, but it is also necessary.

Initial-boundary value problem (1.1) - (1.2) for various elliptic operators $A$ have been considered by a number of authors using different methods (see, for example, handbook [2]). It has been mainly considered the case of the Caputo derivatives $D_{t}^{\rho}$ instead of $\partial_{t}^{\rho}$. In the book of A.A. Kilbas et al. 3 (Chapter 6) there is a survey of works published before 2006. The case of one spatial variable $x \in \mathbb{R}$ and subdiffusion equation with "the elliptical part" $u_{x x}$ were considered for example in the monograph of A. V. Pskhu [1] (Chapter 4, see references thesein). The paper Gorenflo, Luchko and Yamamoto 4] is devoted to the study of subdiffusion equations in Sobelev spaces. In the paper by Kubica and Yamamoto [5], initial-boundary value problems for equations with time-dependent coefficients are considered. In the multidimensional case $\left(x \in \mathbb{R}^{N}\right)$, instead of the differential expression $u_{x x}$, authors considered either the second order elliptic operator (6] - 8]) or elliptic pseudodifferential operators with constant coefficients in the whole space $\mathbb{R}^{N}$ (Umarov 9]). In the paper of Yu. Luchko 7 the author constructed solutions by the eigenfunction expansion in the case of $f=0$ and discussed the unique existence of the generalized solution to problem (1.1) - (1.2) with the Caputo derivative. In his recent paper [ 
A. V. Pskhu considered an initial-boundary value problem for subdiffusion equation with the Laplace operator and domain $\Omega$ - a multidimensional rectangular region. The author succeeded to construct the Green's function. In an arbitrary $N$-dimensional domain $\Omega$ initial-boundary value problems for subdiffusion equations (the fractional part of the equation is a multi-term and initial conditions are non-local) with the Caputo derivatives has been investigated by $\mathrm{M}$. Ruzhansky et al. [10]. The authors proved the existence and uniqueness of the generalized solution to the problem.

A result similar to Theorem 1.2 was obtained in the recent paper 11 for a more general subdiffusion equation. But the conditions on the functions $f(x, t)$ and $\varphi(x)$ that guarantee the existence and uniqueness of the solution to problem (1.1)-(1.2) found in [1] are more stringent. This is due to the fact that in the present paper we give a more precise estimate for the MittagLeffler function $E_{\rho, \rho}(-t), t>0$ (see also [12]).

It is interesting to note that the condition on the function $\varphi(x)$ found in Theorem 1.2 is less restrictive than the analogous condition in the case of an equation (1.1) with derivatives in the sense of Caputo (see, for example, [7, 13]).

\section{Proof of Theorem 1.2}

The uniqueness of the solution can be proved by the standard technique based on completeness in $L_{2}\left(\mathbb{T}^{N}\right)$ of the set of eigenfunctions $\left\{\gamma e^{i n x}\right\}$ (see, for example, [1]).

Proof of existence based on the following lemma (see M.A. Krasnoselski et al. [14], p. 453), which is a simple corollary of the Sobolev embedding theorem.

Lemma 2.1. Let $\sigma>1+\frac{N}{4}$. Then for any $|\alpha| \leq 2$ operator $D^{\alpha}(\hat{A}+1)^{-\sigma}$ (completely) continuously maps from $L_{2}\left(\mathbb{T}^{N}\right)$ into $C\left(\mathbb{T}^{N}\right)$ and moreover the following estimate holds true

$$
\left\|D^{\alpha}(\hat{A}+1)^{-\sigma} g\right\|_{C\left(\mathbb{T}^{N}\right)} \leq C\|g\|_{L_{2}\left(\mathbb{T}^{N}\right)} .
$$

Proof. Since the embedding theorem $\left\|D^{\alpha}(\hat{A}+1)^{-\sigma} g\right\|_{C\left(\mathbb{T}^{N}\right)} \leq C\left\|D^{\alpha}(\hat{A}+1)^{-\sigma} g\right\|_{L_{2}^{a}\left(\mathbb{T}^{N}\right)}$ for $a>N / 2$, then it is sufficient to prove the inequality $\left\|D^{\alpha}(\hat{A}+1)^{-\sigma} g\right\|_{L_{2}^{a}\left(\mathbb{T}^{N}\right)} \leq C\|g\|_{L_{2}\left(\mathbb{T}^{N}\right)}$. But this is a consequence of the estimate

$$
\sum_{n \in \mathbb{Z}^{N}}\left|g_{n}\right|^{2}|n|^{2|\alpha|}(1+A(n))^{-2 \sigma}\left(1+|n|^{2}\right)^{a} \leq C \sum_{n \in \mathbb{Z}^{N}}\left|g_{n}\right|^{2}
$$

that is valid for $\frac{N}{2}<a \leq 2 \sigma-|\alpha|$.

Since the initial condition (1.2) can be rewritten as (see, for example, 1] p. 104)

$$
\lim _{t \rightarrow 0} t^{1-\rho} u(x, t)=\frac{\varphi(x)}{\Gamma(\rho)}
$$

then one can easily verify that the function (1.4) formally satisfies the conditions of problem (1.1)-11.2) (see, for example, 16, p. 173). In order to prove that function (1.4) is actually a solution to the problem, it remains to substantiate this formal statement, i.e. show that the operators $A$ and $\partial_{t}^{\rho}$ can be applied term by term to the series (1.4). To do this we remind the following asymptotic estimate of the Mittag-Leffler function with a sufficiently large negative argument (see, for example, [15], p. 134)

$$
E_{\rho, \rho}(-t)=-\frac{t^{-2}}{\Gamma(-\rho)}+O\left(t^{-3}\right) .
$$

Therefore, since $E_{\rho, \rho}(t)$ is real analytic, one has the estimate

$$
\left|E_{\rho, \rho}(-t)\right| \leq \frac{C}{1+t^{2}}, \quad t>0
$$

We will also use a coarser estimate with a positive $\lambda$ and $0<\varepsilon<1$ :

$$
\left|t^{\rho-1} E_{\rho, \rho}\left(-\lambda t^{\rho}\right)\right| \leq \frac{C t^{\rho-1}}{1+\left(\lambda t^{\rho}\right)^{2}} \leq C \lambda^{\varepsilon-1} t^{\varepsilon \rho-1}, \quad t>0,
$$

which is easy to verify. Indeed, let $t^{\rho} \lambda<1$, then $t<\lambda^{-1 / \rho}$ and

$$
t^{\rho-1}=t^{\rho-\varepsilon \rho} t^{\varepsilon \rho-1}<\lambda^{\varepsilon-1} t^{\varepsilon \rho-1} .
$$


If $t^{\rho} \lambda \geq 1$, then $\lambda^{-1} \leq t^{\rho}$ and

$$
\lambda^{-2} t^{-\rho-1}=\lambda^{-1+\varepsilon} \lambda^{-1-\varepsilon} t^{-\rho-1} \leq \lambda^{\varepsilon-1} t^{\varepsilon \rho-1} .
$$

Note the series (1.4) is in fact the sum of two series. Consider the following partial sums of the first series:

$$
S_{k}^{1}(x, t)=\sum_{|n|^{2}<k} t^{\rho-1} E_{\rho, \rho}\left(-|n|^{2} t^{\rho}\right) \varphi_{n} e^{i n x},
$$

and suppose that function $\varphi$ satisfies the condition of Theorem 1.2 i.e. for some $\tau>\frac{N}{4}$

$$
\sum_{n \in \mathbb{Z}^{N}}|n|^{4(\tau-1)}\left|\varphi_{n}\right|^{2} \leq C_{\varphi}<\infty
$$

Since $\hat{A}^{-\tau-1} e^{i n x}=|n|^{-2(\tau+1)} e^{i n x}$, we may rewrite the sum (2.5) as

$$
S_{k}^{1}(x, t)=\hat{A}^{-\tau-1} \sum_{|n|^{2}<k} t^{\rho-1} E_{\rho, \rho}\left(-|n|^{2} t^{\rho}\right) \varphi_{n}|n|^{2(\tau+1)} e^{i n x} .
$$

Therefore by virtue of Lemma 2.1 one has

$$
\begin{gathered}
\left\|D^{\alpha} S_{k}^{1}\right\|_{C\left(\mathbb{T}^{N}\right)}=\left\|D^{\alpha} \hat{A}^{-\tau-1} \sum_{|n|^{2}<k} t^{\rho-1} E_{\rho, \rho}\left(-|n|^{2} t^{\rho}\right) \varphi_{n}|n|^{2(\tau+1)} e^{i n x}\right\|_{C\left(\mathbb{T}^{N}\right)} \leq \\
\leq C\left\|\sum_{|n|^{2}<k} t^{\rho-1} E_{\rho, \rho}\left(-|n|^{2} t^{\rho}\right) \varphi_{n}|n|^{2(\tau+1)} e^{i n x}\right\|_{L_{2}\left(\mathbb{T}^{N}\right)} .
\end{gathered}
$$

Using the orthonormality of the system $\left\{e^{i n x}\right\}$, we will have

$$
\left\|D^{\alpha} S_{k}^{1}\right\|_{C\left(\mathbb{T}^{N}\right)}^{2} \leq\left.\left. C \sum_{|n|^{2}<k}\left|t^{\rho-1} E_{\rho, \rho}\left(-|n|^{2} t^{\rho}\right) \varphi_{n}\right| n\right|^{2(\tau+1)}\right|^{2} .
$$

Application of estimate (2.3) and inequality $\left(|n|^{2} t^{\rho}\right)^{2}\left(1+\left(|n|^{2} t^{\rho}\right)^{2}\right)^{-1}<1$ gives

$$
\left.\left.\sum_{|n|^{2}<k}\left|t^{\rho-1} E_{\rho, \rho}\left(-|n|^{2} t^{\rho}\right) \varphi_{n}\right| n\right|^{2(\tau+1)}\right|^{2} \leq C t^{-2(\rho+1)} \sum_{|n|^{2}<k}|n|^{4(\tau-1)}\left|\varphi_{n}\right|^{2} \leq C t^{-2(\rho+1)} C_{\varphi} .
$$

Therefore we can rewrite the estimate (2.8) as

$$
\left\|D^{\alpha} S_{k}^{1}\right\|_{C\left(\mathbb{T}^{N}\right)}^{2} \leq C t^{-2(\rho+1)} C_{\varphi} .
$$

This implies uniformly on $x \in \mathbb{T}^{N}$ convergence of the differentiated sum (2.5) with respect to the variables $x_{j}$ for each $t \in(0, T]$. On the other hand, the sum (2.6) converges for any permutation of its members as well, since these terms are mutually orthogonal. This implies the absolute convergence of the differentiated sum (2.5) on the same interval $t \in(0, T]$.

Now we consider the second part of the series (1.4):

$$
S_{k}^{2}(x, t)=\sum_{|n|^{2}<k} \int_{0}^{t} f_{n}(t-\xi) \xi^{\rho-1} E_{\rho, \rho}\left(-|n|^{2} \xi^{\rho}\right) d \xi e^{i n x}
$$

and suppose that function $f(x, t)$ satisfies all the conditions of Theorem 1.2, i.e. the following series converges uniformly on $t \in[0, T]$ for some $\tau>\frac{N}{4}$ :

$$
\sum_{n \in \mathbb{Z}^{N}} t^{2(1-\rho)}|n|^{4 \tau}\left|f_{n}(t)\right|^{2} \leq C_{f}<\infty .
$$

We choose a small $\varepsilon>0$ in such a way, that $\tau+1-\varepsilon>1+\frac{N}{4}$. Since $\hat{A}^{-\tau-1+\varepsilon} e^{i n x}=$ $|n|^{-2(\tau+1-\varepsilon)} e^{i n x}$, we may rewrite the sum (2.8) as

$$
S_{k}^{2}(x, t)=\hat{A}^{-\tau-1+\varepsilon} \sum_{|n|^{2}<k} \int_{0}^{t} f_{n}(t-\xi) \xi^{\rho-1} E_{\rho, \rho}\left(-|n|^{2} \xi^{\rho}\right) d \xi|n|^{2(\tau+1-\varepsilon)} e^{i n x} .
$$

Then by virtue of Lemma 2.1 one has

$$
\left\|D^{\alpha} S_{k}^{2}\right\|_{C\left(\mathbb{T}^{N}\right)}=\left\|D^{\alpha} \hat{A}^{-\tau-1+\varepsilon} \sum_{|n|^{2}<k} \int_{0}^{t} f_{n}(t-\xi) \xi^{\rho-1} E_{\rho, \rho}\left(-|n|^{2} \xi^{\rho}\right) d \xi|n|^{2(\tau+1-\varepsilon)} e^{i n x}\right\|_{C\left(\mathbb{T}^{N}\right)} \leq
$$




$$
\leq C\left\|\sum_{|n|^{2}<k} \int_{0}^{t} f_{n}(t-\xi) \xi^{\rho-1} E_{\rho, \rho}\left(-|n|^{2} \xi^{\rho}\right) d \xi|n|^{2(\tau+1-\varepsilon)} e^{i n x}\right\|_{L_{2}\left(\mathbb{T}^{N}\right)} .
$$

Using the orthonormality of the system $\left\{e^{i n x}\right\}$, we will have

$$
\left\|D^{\alpha} S_{k}^{2}\right\|_{C\left(\mathbb{T}^{N}\right)}^{2} \leq\left.\left. C \sum_{|n|^{2}<k}\left|\int_{0}^{t} f_{n}(t-\xi) \xi^{\rho-1} E_{\rho, \rho}\left(-|n|^{2} \xi^{\rho}\right) d \xi\right| n\right|^{2(\tau+1-\varepsilon)}\right|^{2} .
$$

Now we use estimate (2.4) and apply the generalized Minkowski inequality. Then

$$
\left\|D^{\alpha} S_{k}^{2}\right\|_{C\left(\mathbb{T}^{N}\right)}^{2} \leq C\left(\int_{0}^{t} \xi^{\varepsilon \rho-1}(t-\xi)^{\rho-1}\left(\sum_{|n|^{2}<k}|n|^{4 \tau}(t-\xi)^{2(1-\rho)}\left|f_{n}(t-\xi)\right|^{2}\right)^{1 / 2} d \xi\right)^{2} \leq C \cdot C_{f},
$$

where $C$ depends on $T$ and $\varepsilon$. Hence, using the same argument as above, we see that the differentiated sum (2.8) with respect to the variables $x_{j}$ converges absolutely and uniformly on $(x, t) \in \mathbb{T}^{N} \times[0, T]$.

Further, from equation (1.1) one has

$$
\partial_{t}^{\rho}\left(S_{k}^{1}+S_{k}^{2}\right)=-A\left(S_{k}^{1}+S_{k}^{2}\right)+\sum_{|n|^{2}<k} f_{n}(t) e^{i n x} .
$$

Absolutely and uniformly convergence of the latter series can be proved as above.

Thus Theorem 1.2 is completely proved.

\section{ACKNOWLEDGEMEnT}

The authors convey thanks to Sh. A. Alimov for discussions of these results.

\section{REFERENCES}

1. Pskhu, A.V.: Fractional partial differential equations (in Russian), M. NAUKA (2005)

2. Kochubei A., Luchko Yu.. Handbook of Fractional Calculus with Applications. Volume 2: Fractional Differential Equations. De Gruyter 2019.

3. Kilbas, A.A., Srivastava, H.M., Trujillo, J.J.: Theory and applications of fractional differential tquations, ELSEVIER (2006)

4. R. Gorenflo, Yu. Luchko, M. Yamamoto, Time-fractional diffusion equation in the fractional Sobolev spaces, Fract. Calc. Appl. Anal. 18, 799-820 (2015).

5. A. Kubica, M. Yamamoto, Initial-boundary value problems for fractional diffusion equations with time-dependent coefficients, Fract. Calc. Appl. Anal. 21, 276-311 (2018).

6. Agrawal, O. P.: Solution for a fractional diffusion-wave equation defined in a bounded domain, Nonlin. Dynam. 29, 145-155 (2002)

7. Yu. Luchko, Some uniqueness and existence results for the initial-boundary value problems for the generalized time-fractional diffusion equation, Comput. Math. Appl. 59 (2010) 1766-1772.

8. Pskhu, A.V.: Green function of the first doundary-value problem for the fractional diffusion wave equation in a multidimentiona rectangular domain, Achievements of sciens and technology. Modern mathematics and its applications (in Russian), 167, 52-61 (2019)

9. Umarov, S.: Introduction to fractional and pseudo-differential equations with singular symbols, Springer (2015)

10. Ruzhansky, M., Tokmagambetov, N., Torebek, B.T.: On non-local problem for a multi-term fractional diffusion-wave equation, arXiv:1812.01336 2 [math. AP] 5 Dec (2018)

11. R. Ashurov, O. Muhiddinova, Initial-boundary value problem for a time-fractional subdiffusion equation with an arbitrary elliptic differential operator, Lobachevski Journal of Mathematics, 2021, Vol. 42, No. 3, pp. 517-525.

12. Alimov Sh.A., Ashurov R.R. On the backward problems in time for time-fractional subdiffusion equations. //https://arxiv.org/pdf/2105.06258.pdf

13. K. Sakamoto and M. Yamamoto, Initial value/boundary value problems for fractional diffusion-wave equations and applications to some inverse problems, J. Math. Anal. Appl. 382 (2011) 426-447.

14. Krasnoselski, M.A., Zabreyko, P.P., Pustilnik E.I., Sobolevski, P.S.: Integral operators in the spaces of integrable functions (in Russian), M. NAUKA (1966)

15. M. M. Dzherbashian [=Djrbashian], Integral Transforms and Representation of Functions in the Complex Domain (in Russian), M. NAUKA, 1966. 
16. Gorenflo, R., Kilbas, A.A., Mainardi, F., Rogozin, S.V.: Mittag-Leffler functions, related topics and applications, Springer (2014)

17. Alimov, Sh.A., Ashurov, R.R., Pulatov, A.K.: Multiple Fourier Series and Fourier Integrals. Commutative Harmonic Analysis, Springer, Berlin (1992)

National University of Uzbekistan named after Mirzo Ulugbek and Institute of Mathematics, Uzbekistan ACAdemy of Science

Current address: Institute of Mathematics, Uzbekistan Academy of Science, Tashkent, 81 Mirzo Ulugbek str. 100170

Email address: ashurovr@gmail.com 